\title{
Laser Capture Microdissection of Single Neurons with Morphological Visualization Using Fluorescent Proteins Fused to Transmembrane Proteins
}

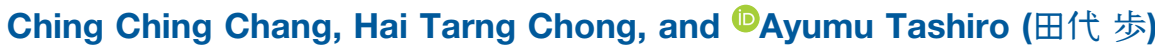

https://doi.org/10.1523/ENEURO.0275-20.2021

School of Biological Sciences, Nanyang Technological University, Singapore 308232

\begin{abstract}
Gene expression analysis in individual neuronal types helps in understanding brain function. Genetic methods expressing fluorescent proteins are widely used to label specific neuronal populations. However, because cell type specificity of genetic labeling is often limited, it is advantageous to combine genetic labeling with additional methods to select specific cell/neuronal types. Laser capture microdissection is one of such techniques with which one can select a specific cell/neuronal population based on morphological observation. However, a major issue is the disappearance of fluorescence signals during the tissue processing that is required for highquality sample preparation. Here, we developed a simple, novel method in which fluorescence signals are preserved. We use genetic labeling with fluorescence proteins fused to transmembrane proteins, which shows highly stable fluorescence retention and allows for the selection of fluorescent neurons/cells based on morphology. Using this method in mice, we laser-captured neuronal somata and successfully isolated RNA. We determined that $\sim 100$ cells are sufficient to obtain a sample required for downstream applications such as quantitative PCR. Capability to specifically microdissect targeted neurons was demonstrated by an $\sim 10$-fold increase in mRNA for fluorescent proteins in visually identified neurons expressing the fluorescent proteins compared with neighboring cells not expressing it. We applied this method to validate virus-mediated singlecell knockout, which showed up to $92 \%$ reduction in knocked-out gene RNA compared with wild-type neurons. This method using fluorescent proteins fused to transmembrane proteins provides a new, simple solution to perform gene expression analysis in sparsely labeled neuronal/cellular populations, which is especially advantageous when genetic labeling has limited specificity.
\end{abstract}

Key words: channelrhodopsin; gene expression; halorhodopsin; optogenetics; viral vector

\section{Significance Statement}

Genetic labeling of specific cell types with reporter fluorescent proteins is widely used for gene expression analysis. However, because the specificity of genetic labeling is often limited, an additional method is required to collect samples from a specific cell type. Here, we developed a novel method to allow for the selection of a cell/neuronal type of interest based on morphology before performing sample collection using laser capture microdissection. Stable fluorescent signals from fluorescent proteins fused to transmembrane proteins allow us to morphologically select and laser capture hundreds of single neurons. This method would be applicable to genomic and transcriptomic analysis of different cell/neuronal types and could facilitate our understanding of brain functions.

Received June 23, 2020; accepted July 15, 2021; First published August 16, 2021.

The authors declare no competing financial interests.
Author contributions: C.C.C. and A.T. designed research; C.C.C. and H.T.C. performed research; C.C.C. and A.T. analyzed data; C.C.C. and A.T. wrote the paper. 


\section{Introduction}

Gene expression analysis of specific neuronal types has been successfully performed using genetic labeling with reporter fluorescent proteins and fluorescenceactivated cell sorting (FACS; Xia et al., 2017; Daigle et al., 2018). However, one technical limitation is that genetic labeling often marks multiple neuronal and/or other cell types (van Praag et al., 2002; Balthasar et al., 2004; Lagace et al., 2007; Harris et al., 2014), whereas FACS cannot separate such unwanted, labeled cell types. In addition, enzyme-mediated cell dissociation procedures could affect viability and gene expression (Ho et al., 2018; Hussain et al., 2018; Reichard and Asosingh, 2019). An alternative method that allows for separating cell/neuronal types without tissue dissociation steps would be useful as a complementary method.

Laser capture microdissection is a technique that dissects a small piece of tissue or a single cell by a laser and collects them with gravity or the assistance of a laser. Instruments for laser capture microdissection are often equipped with a fluorescence light source (Emmert-Buck et al., 1996; Bonner et al., 1997; Fend and Raffeld, 2000). Therefore, one can observe the morphology of genetically labeled fluorescent cells and select a cell type of interest to capture. However, in practice, this is not a simple task. Commonly for laser capture microdissection, sections are prepared from freshly frozen tissue, rather than fixed tissue, to acquire high-quality RNA. In such sections, fluorescent proteins diffuse out from cells and their fluorescence signals disappear during tissue-processing procedures (Singh et al., 2019). Although an optimized protocol can improve the issue to some extent, the instability of fluorescence signals makes it difficult to use fluorescence as a guide to distinguish cell types for laser capture microdissection. A more stable fluorescence-labeling method would be valuable for solving this technical limitation. In this line, studies (Rossner et al., 2006; Chatzi et al., 2019) demonstrated that nuclear targeted fluorescent proteins preserves their signals. However, because only nuclei, but not processes/neurites, are visualized, their application to morphological identification of cell types is limited.

In the course of an experiment for some other purpose, we happened to find that the fluorescence signals from transmembrane proteins fused with fluorescent proteins were extremely stable and kept visualizing dendritic morphology in air-dried, nonfixed brain sections. We later found that similar preservation of fluorescence signals from transmembrane proteins fused with fluorescent proteins has been previously described in the context of flow

This research was supported by the Ministry of Education, Singapore, Academic Research Fund Tier 1 (Grants 2016-T1-001-010, 2018-T1-002-053) and Tier 3 (Grant MOE2017-T3-1-002).

Acknowledgements: We thank Miaomiao Han for technical assistance.

Correspondence should be addressed to Ayumu Tashiro at atashiro@ ntu.edu.sg or Ching Ching Chang at chingchingchang65@gmail.com.

https://doi.org/10.1523/ENEURO.0275-20.2021 Copyright $@ 2021$ Chang et al.

This is an open-access article distributed under the terms of the Creative Commons Attribution 4.0 International license, which permits unrestricted use, distribution and reproduction in any medium provided that the original work is properly attributed. cytometry of primary human fibroblasts (Kalejta et al., 1997). This observation made us reason that genetic labeling with fluorescent proteins fused to transmembrane proteins allows us to identify a cell type of interest based on their morphology and selectively capture them using laser capture microdissection. In this study, we tested this possibility and validated that the use of labeling with fluorescent proteins fused to transmembrane proteins allows us to select neurons over non-neuronal cells and perform downstream gene expression analyses.

\section{Materials and Methods}

A flowchart of the methodology is shown in Figure 1. We freshly froze brains after retrieving them from the skull. We sectioned the brains, mounted sections on the glass slides and dehydrated/fixed them with ethanol. We distinguished neurons from glial cells based on their dendritic morphology under a fluorescence microscope, and then harvested the cell bodies of the selected neurons with laser capture microdissection. A total of 100-300 microdissected neurons were pooled for RNA extraction. As examples of downstream gene expression analyses, we performed quantitative PCR (qPCR) analysis after cDNA amplification.

\section{Mice}

We used 6- to 11-week-old C57BL/6 or transgenic mice of either sex, housed in a $12 \mathrm{~h}$ dark/light cycle with ad libitum access to water and food. Transgenic lines used were floxed NR1 mice (B6.129S4-Grin1 ${ }^{\text {tm2Stl/J; }}$ Tsien et al., 1996) and Pomc-ChR2 mice, which were produced by crossing two transgenic lines: STOCK Tg(Pomc1-cre)16Lowl/J (back-crossed with C57BL/6 mice; Balthasar et al., 2004) and B6.Cg-Gt(ROSA)26Sortm32(CAG-COP4*H134R/EYFP) $\mathrm{Hze} / \mathrm{J}$ (Madisen et al., 2012). All three transgenic lines were originally purchased from The Jackson Laboratory and bred in our local facilities. All animal procedures were performed according to the approval from the Institutional Animal Care and Use Committee at Nanyang Technological University.

\section{Preparation of brain sections and tissue processing}

Mice were deeply anesthetized with isoflurane. Mice were decapitated, and their brains were dissected out from the skull within $2 \mathrm{~min}$. The brains were transferred onto an embedding mold and frozen in embedding medium (O.C.T. Compound, Sakura Finetek) with isopentane on dry ice, which was completed within an additional 1-3 min. Brains were stored at $-80^{\circ} \mathrm{C}$ until cryosectioning. Immediately before cryosectioning, brains were kept at $-20^{\circ} \mathrm{C}$ for at least $30 \mathrm{~min}$. The $10-$ to $12-\mu \mathrm{m}-$ thick sections were produced using a cryostat microtome at $-20^{\circ} \mathrm{C}$. Brain sections were mounted on glass slides and air dried for $5 \mathrm{~min}$. Sections were dehydrated/fixed in increasing concentrations of ethanol (75\%, 95\%, and then 100\%) for 30 s each, followed by xylene for $1 \mathrm{~min}$. We performed these procedures immediately before fluorescence visualization and laser capture microdissection. 


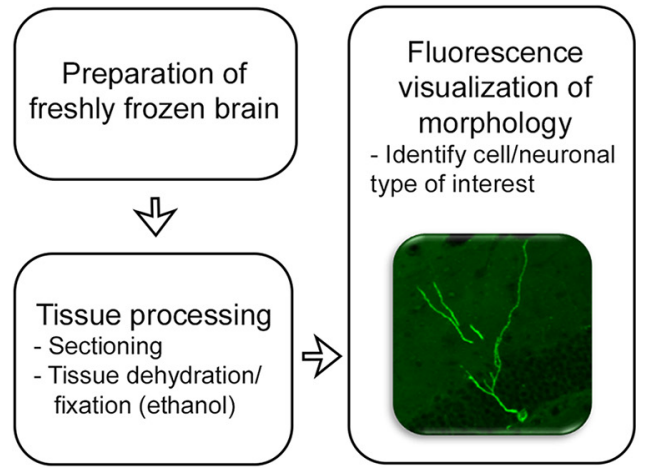

Figure 1. Flowchart of methodology.

\section{Fluorescence visualization and laser capture microdissection}

We used the PALM MicroBeam System (Zeiss) equipped with a fluorescence microscope. Each fluorescently labeled cell was selected under the microscope with an LD PlanNeofluar objective lens $(40 \times / 0.6$ numerical aperture), and laser captured into an AdhesiveCap 500 opaque (Zeiss). A sample of up to $\sim 100$ cells were collected in a single AdhesiveCap and lysed with cell lysis buffer. When the collection took more than $1 \mathrm{~h}$, we lysed cells to minimize sample degradation and continued collection in a new AdhesiveCap.

\section{RNA extraction}

After cell lysis in the AdhesiveCaps, we extracted RNA using an Ambion RNA Isolation Kit (Thermo Fisher Scientific) according to the manufacturer protocol. When more than one Adhesivecap was used for sample collection, cell lysates from multiple AdhesiveCaps were pooled and passed through a single column. RNA concentration was measured using Quant-iT RiboGreen (Thermo Fisher Scientific).

\section{Quantitative PCR}

Reverse transcription and cDNA amplification were performed using cDNA SMARTer PCR cDNA Synthesis Kit (Takara Bio) or GeneChip WT Pico Kit (Affymetrix). qPCR was performed with iTaq Universal SYBR Green Supermix (Bio-Rad) and StepOnePlus Real-Time PCR System (Thermo Fisher Scientific), using the following gene-specific primers: NR1 forward, TGTCCTGGGACTGACTACCC; NR1 reverse, CTGACCAGCAGGATGA; yellow fluorescent protein (YFP) forward, TATATCATGGCCGACAAGCA; YFP reverse, TGTTCTGCTGGTAGTGGTCG; doublecortin (DCX) forward, GAGTGCGCTACATTTATACCATTG; DCX reverse, TGACATTCTTGGTGTACTCAACCT; CaMKIl $\alpha$ forward, AG GATGAAGACACCAAAGTGC; CaMKIl $\alpha$ reverse, GGTTC AAAGGCTGTCATTCC; $\beta$-actin forward, AGCCATGTAC GTAGCCATCC; and $\beta$-actin reverse, CTCTCAGCTGTGGT GGTGAA. Each sample was amplified in triplicate. We used mouse liver total DNA (see Fig. 4D) that was included in cDNA SMARTer PCR cDNA Synthesis Kit (Takara Bio).

\section{RNA integrity assay}

The quality of RNA samples that we isolated was evaluated by a service provided by NovogeneAIT Genomics

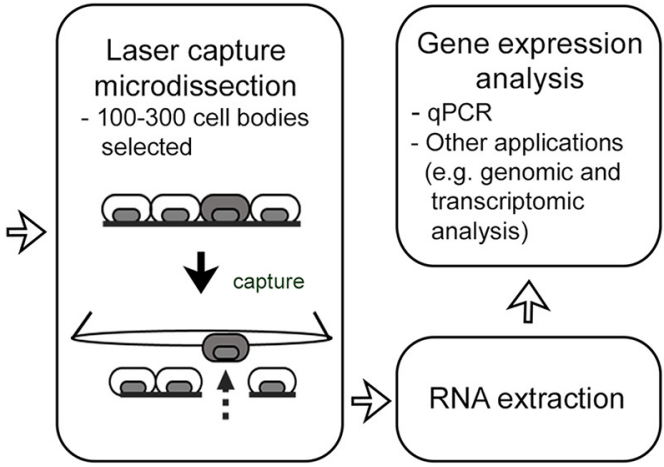

Singapore Pte Ltd, using an Agilent RNA 6000 Pico Kit and an Agilent 2100 Bioanalyzer. The quality of RNA was determined as the RNA integrity number (Schroeder et al., 2006).

\section{Preparation of viral vectors}

We prepared the viral vectors as previously described (Tashiro et al., 2015a). We used Moloney murine leukemia virus-based retroviral vectors driving the expression of eNpHR3.0-YFP, mCherry, GFP-F, ChR2(SFFO), or bicistronically eNPHR3.0YFP and Cre under the control of the CAG promoter (Zhao et al., 2006) and an adeno-associated viral vector expressing mCherry-TRPV2 (transient receptor potential cation channel V2) under the control of the CaMKII $\alpha$ promoter (Kitanishi et al., 2015).

\section{Stereotaxic injections}

Mice were bilaterally injected with retroviral vectors $(1.5 \mu$, titer: $1 \times 10^{7}$ to $1 \times 10^{8}$ colony-forming units $/ \mathrm{ml}$ ) into the dentate gyrus (coordinates: anteroposterior, $2 \mathrm{~mm}$ from the bregma; mediolateral, $1.5 \mathrm{~mm}$ from the bregma; dorsoventral, 2.3 $\mathrm{mm}$ from skull surface). Preparation and injection procedures were described previously (Tashiro et al., 2015a). For coinjection, a mixture of virus solutions was prepared with a 1:1 ratio in volume.

\section{Perfusion and fixation}

For the purpose of visualizing fluorescence expression in neurons (Fig. 2, see also Fig. 7C), brain sections were prepared from brains that were perfusion fixed with $4 \%$ paraformaldehyde. The procedure was previously described (Tashiro et al., 2015b). Brain sectioning was performed in the same way as described in the subsection Preparation of brain sections and tissue processing. Sections were mounted on glass slides with coverslips and antifade polyvinyl alcohol mounting medium with DABCO.

\section{Freeze-thaw cycle with HEK293 cell culture with or without cryoprotectant}

We seeded $1 \times 10^{5}$ HEK293 cells in wells in a 12-well plate. Next day, we transfected the cells with one of the plasmids listed below, with a calcium phosphate method with the following mixture: $500 \mathrm{ng}$ of plasmid DNA in 
A

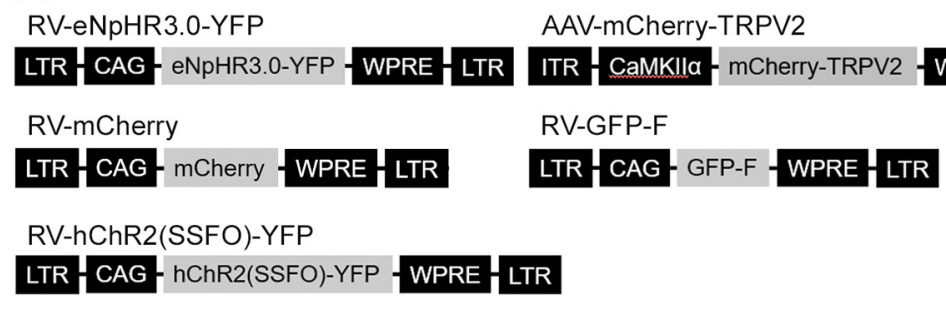

B
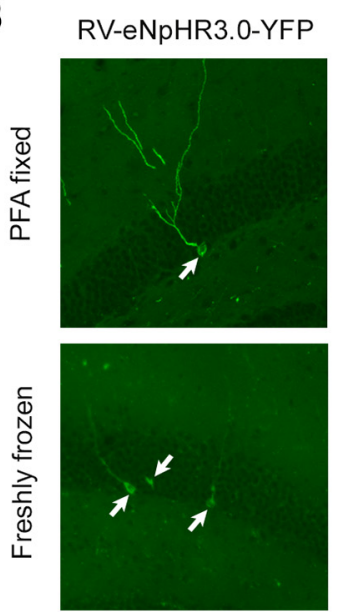
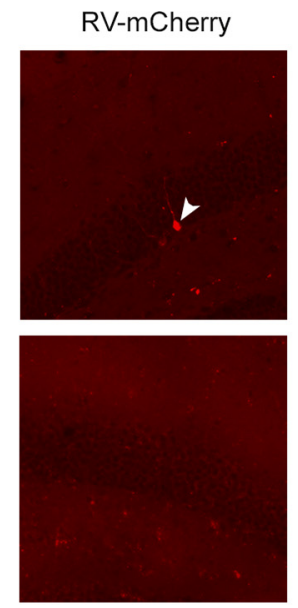

D

RV-hChR2(SSFO)-YFP

$E$
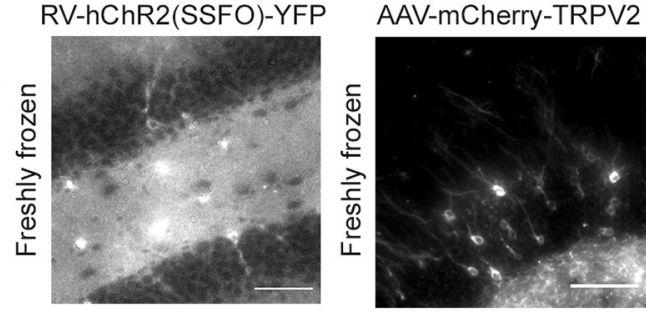

C Transgenic mouse
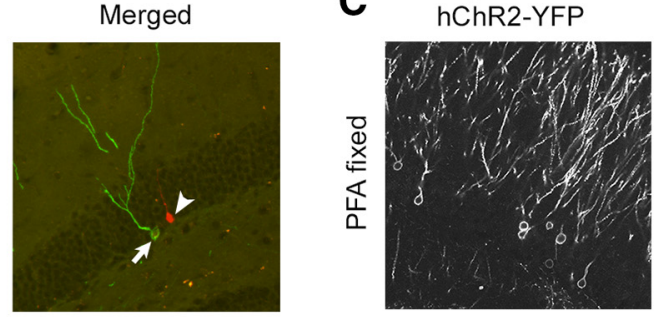

$\mathbf{F}$
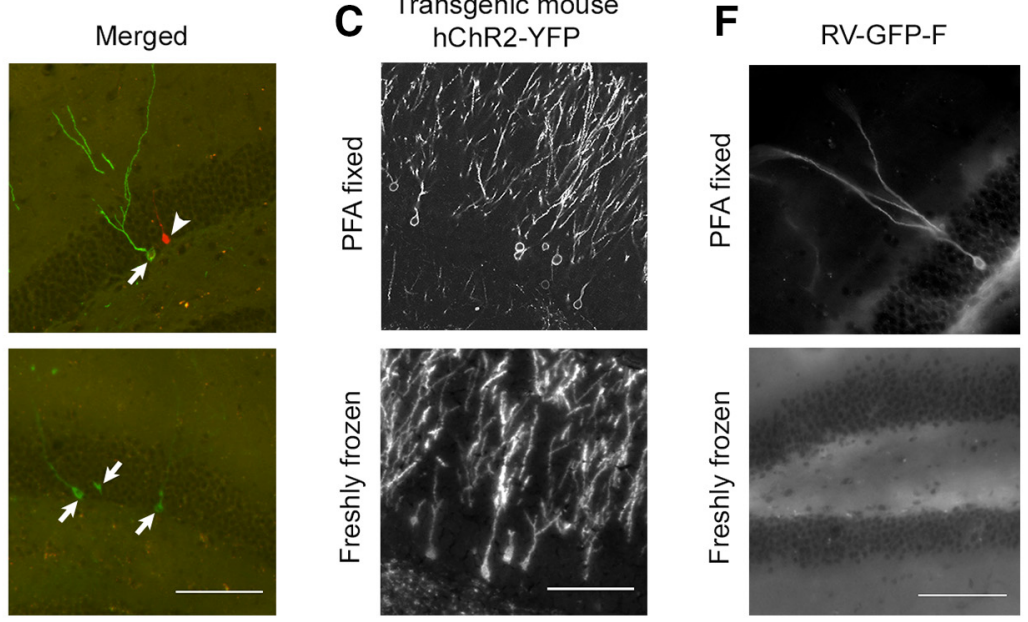

Figure 2. The signals of fluorescent proteins fused to transmembrane proteins are preserved after the standard tissue-processing procedure for laser capture microdissection. $\boldsymbol{A}$, Schematic structures of the viral vector genomes. $\boldsymbol{B}-\boldsymbol{F}$, Images of fluorescently labeled granule cells in the dentate gyrus of mice injected with a mixture of two retroviral vectors expressing YFP-fused enhanced halorhodopsin, eNpHR3.0-YFP, or mCherry (B); Pomc-ChR2-YFP transgenic mice $(\boldsymbol{C})$ and mice injected with a retroviral vector expressing ChR2(SSFO)YFP $(\boldsymbol{D})$; an adeno-associated viral vector expressing mCherry-TRPV2 $(\boldsymbol{E})$ and a retroviral vector expressing a membrane-targeted form of GFP, GFP-F (F). Brain sections were prepared from paraformaldehyde fixed or freshly frozen brains and went through the standard tissueprocessing procedure. Note that fluorescence signals of eNpHR3.0-YFP (B), ChR2-YFP (C), ChR2(SSFO)-YFP (D), and mCherry-TRPV2 (E), but not mCherry $(\boldsymbol{B})$ and GFP-F $(\boldsymbol{F})$, were preserved in sections prepared from freshly frozen brains. Arrows and arrowheads in $\boldsymbol{B}$ indicate somata labeled by eNpHR3.0-YFP and mCherry. RV, Retroviral vector; AAV, adeno-associated viral vector; LTR, long-terminal repeat; CAG, CAG promoter; WPRE, Woodchuck hepatitis virus post-transcriptional regulatory element; CaMKII $\alpha$, the CaMKII $\alpha$ promoter; ITR, inverted terminal repeat. Scale bars, $100 \mu \mathrm{m}$.

$640 \mu$ of sterile Milli-Q water and $36 \mu$ of $2 \mathrm{M} \mathrm{CaCl}_{2}$, with $100 \mu \mathrm{l}$ of $2 \times$ HEBS $(50 \mathrm{mM}$ HEPES, $280 \mathrm{mM} \mathrm{NaCl}, 10 \mathrm{mM}$ $\mathrm{KCl}, 1.5 \mathrm{mM} \mathrm{Na}_{2} \mathrm{HPO}_{4}, 12 \mathrm{mM}$ D-glucose, $\mathrm{pH}$ 7.05). The plasmid DNAs were retroviral transfer plasmids, RV-CAGGFP, RV-CAG-mCherry, RV-CAG-eNpHR3.0-YFP, RVCAG-ChR2-mCherry, and RV-CAG-ChR2(SSFO)-YFP.

Twenty-four hours after transfection, we removed culture media from the wells and washed twice with PBS. For cells with cryoprotectant, we applied $500 \mu \mathrm{l}$ of $10 \%$ $\mathrm{DMSO} / 90 \%$ fetal bovine serum on top of the cells before a freeze-thaw cycle. For cells without cryoprotectant, we air dried and froze/thawed cells without cryoprotectant. We froze the cells by keeping the 12 -well plate in a $-80^{\circ} \mathrm{C}$ freezer for $30 \mathrm{~min}$. We then thawed them at room temperature and performed imaging immediately.

\section{Results}

\section{Fluorescence signals from fluorescent proteins fused to transmembrane proteins are preserved after tissue processing}

In preliminary experiments, we tried to laser capture single neurons labeled with regular fluorescent proteins, such as the green fluorescent protein (GFP), which is soluble in the cytoplasm. After tissue-processing procedures, fluorescence signals disappeared in sections prepared from freshly frozen brains, and our attempt failed. However, in the course of an experiment for some other purpose, we happened to find that fluorescence signals from fluorescent proteins fused to transmembrane proteins are extremely stable even in air-dried, nonfixed brain sections prepared from freshly frozen brains. Under the same conditions, the fluorescence signals of regular fluorescent proteins diffuse and dissipate quickly in the order of seconds to minutes. This preliminary finding led us to test whether labeling with fluorescent proteins fused to transmembrane proteins is preserved after the standard tissue-processing procedure for laser capture microdissection.

Figure 2 illustrates the instability of cytoplasmic fluorescent proteins and the stability of fluorescent proteins fused to transmembrane proteins in sections from freshly frozen brains, which we processed by the standard protocol of tissue processing used for laser capture microdissection. We injected a mixture of two retroviral vectors 
A
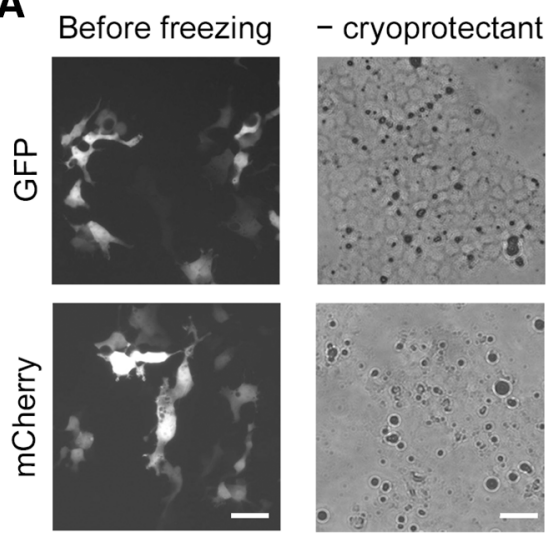

\section{B}

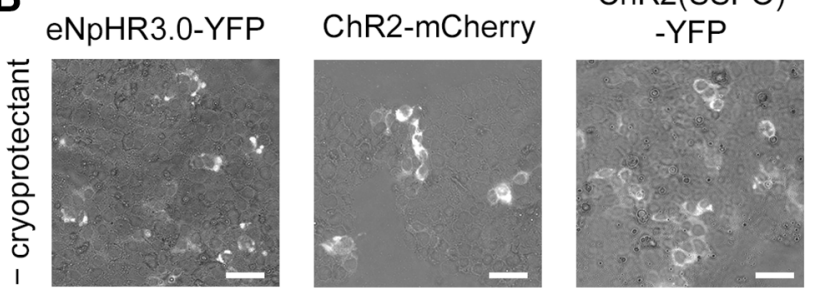

Figure 3. Cryoprotectant prevents the loss of fluorescence signals from regular fluorescent proteins in HEK293 cell culture after a freeze-thaw cycle. $\boldsymbol{A}$, Representative images showing fluorescence signals from regular, cytosolic fluorescent proteins (GFP and mCherry) after a freeze-thaw cycle with or without cryoprotectant. Fluorescence signals disappeared without cryoprotectant while cryoprotectant preserved fluorescence signals. $\boldsymbol{B}$, Representative images showing fluorescence signals from fluorescent proteins fused to transmembrane proteins [eNpHR3.0-YFP, ChR2-mCherry, ChR2 (SSFO)-YFP]. Fluorescence signals were preserved without cryoprotectant after a freeze-thaw cycle. Scale bar, $50 \mu \mathrm{m}$.

into the dentate gyrus of adult mice (Fig. 2A). One of them was for expressing YFP fused to transmembrane proteins (enhanced halorhodopsin, eNpHR3.0), while the other was for expressing mCherry, which is cytosolic fluorescent proteins. Some of the mice were perfusion fixed with $4 \%$ paraformaldehyde, while the brains of the other mice were freshly frozen without fixation. All brains were then subjected to the standard tissue-processing procedure. As expected, paraformaldehyde-fixed brain sections preserved both YFP and mCherry signals (Fig. 2B, top). On the other hand, in sections from freshly frozen brains, YFP signals were preserved while mCherry signals were completely lost (Fig. 2B, bottom). Thus, fluorescent proteins fused to transmembrane proteins, but not cytosolic fluorescent proteins, preserve the fluorescence signals after the standard tissue-processing procedure for laser capture microdissection. Importantly, the dendritic morphology of $\mathrm{YFP}^{+}$neurons was preserved and clearly visualized in the sections from freshly frozen brains (Fig. 2B).

In addition to enhanced halorhodopsin, we tested fluorescent proteins fused to another type of transmembrane proteins, channelrhodopsin-2 (ChR2). We prepared brain sections from (1) Pomc-ChR2-YFP mice expressing ChR2 fused to YFP in a subset of granule cells in the dentate gyrus and (2) adult mice that were injected with retroviral vector expressing a stable step function opsin (SSFO) mutant of ChR2 fused to YFP (Fig. 2A) into the dentate gyrus. Then we performed tissue processing. As shown in Figure 2, $C$ and $D$, fluorescence signals in neurons were preserved in freshly frozen sections.

ChR2 and eNpHR3.0 are structurally similar proteins. To test whether fusion to a different type of transmembrane proteins also preserves fluorescence signals, we prepared brain sections from mice injected with an adeno-associated viral vector expressing TRPV2 (Fig. 2A) into the dentate gyrus. As shown in Figure 2E, fluorescence signals were again preserved in freshly frozen sections.

To test whether membrane-targeted fluorescent proteins with a farnesylation signal are preserved after tissue processing, we injected a retroviral vector expressing GFP fused with a farnesylation signal (GFP-F; Moriyoshi et al., 1996; Jiang and Hunter, 1998; Fig. 2A) into the dentate gyrus of adult mice. In cells, a farnesyl group is added to GFP-F and facilitates the attachment to cellular membrane. Although sections from brains fixed with paraformaldehyde preserved clear fluorescence labeling of neurons (Fig. $2 F$, top), no fluorescence signal was detected in the sections from freshly frozen brains (Fig. $2 F$, bottom). Thus, membrane-targeted fluorescence proteins do not preserve its fluorescence signals through the standard tissue-processing procedure.

We found that the loss of fluorescence signals from regular fluorescent proteins also occurs in HEK293 cells after freezing and thawing (Fig. $3 A$ ). However, when we added cryoprotectant (10\% DMSO/90\% fetal bovine serum), fluorescence signals were retained. This is presumably because cryoprotectant prevented the freeze-thaw cycle from rupturing the plasma membrane. In contrast, fluorescence signals from fluorescent proteins fused to transmembrane proteins were preserved after a freeze-thaw cycle without cryoprotectant (Fig. 3B). The results suggest that the breakage of plasma membrane caused by freezing causes the leakage of regular cytoplasmic fluorescent proteins but still keeps fluorescent proteins fused to transmembrane proteins in HEK293 cells, which is likely the same in brain sections.

\section{Laser capture microdissection of morphologically identified neurons}

Next, we performed laser capture microdissection of single neurons labeled with fluorescent proteins fused to transmembrane proteins. We injected a retroviral vector expressing eNpHR3.0-YFP into adult mice (Fig. $4 A$ ) and, after $14 \mathrm{~d}$, harvested their brains and prepared brain sections using the standard tissue-processing protocol. When injected into the dentate gyrus of adult mice, the type of retroviral vectors we used in this study are known to mainly transduce in newly generated neurons in the dentate gyrus (Fig. 2B). However, a modest number of non-neuronal cells are also infected to express transgenes. Under a fluorescence microscope, we were able to distinguish neurons from non-neuronal cells based on 
A

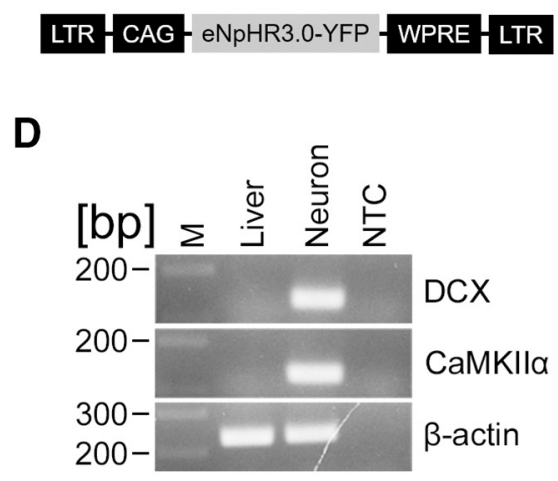

B
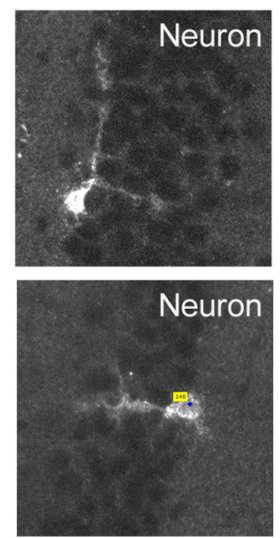

C
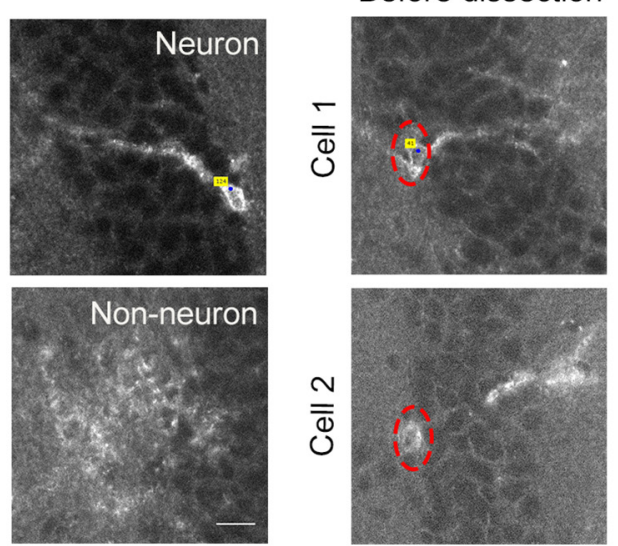

After dissection
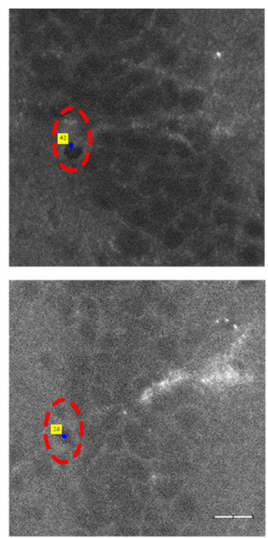

Figure 4. Laser capture microdissection of fluorescence labeled neurons. A, A schematic structure of the viral vector genome. B, Images of eNpHR3.0-YFP-expressing neurons and non-neuronal cells in the dentate gyrus of mice that were injected with a retroviral vector expressing eNpHR3.0-YFP. C, Fluorescence images of sections before and after laser capture microdissection. Red dotted ovals show the location of somata of fluorescently labeled neurons selected for laser capture microdissection. Blue dots with yellow labels indicate the center of a selected area used for laser capture. Scale bar, $20 \mu \mathrm{m}$. $\boldsymbol{D}$, An electrophoresis image showing PCR amplification end products for the $\mathrm{DCX}, \mathrm{CaMKIl} \alpha$, and $\beta$-actin genes. Note the high expression of neuronal markers in fluorescently labeled neurons compared with a total liver RNA sample where DCX and CaMKIl $\alpha$ were not detected. M, Molecular marker; NTC, nontemplate control.

their morphology (Fig. 4B). The somata of labeled neurons were quickly identified and laser captured before being photo-bleached by excitation light. Figure $4 \mathrm{C}$ shows fluorescence images from sections before and after laser capture microdissection, which demonstrates the specific removal of the fluorescence signals in the somata of single neurons while maintaining surrounding tissue intact.

\section{Successful cDNA preparation and qPCR analysis}

We pooled 150 and 300 laser-captured, single neuronal somata and extracted RNA. From these neuronal somata, we acquired 350 and $900 \mathrm{pg}$ of RNA samples, respectively. These yields of RNA are sufficient for reverse transcription and cDNA amplification using the GeneChip WT Pico Kit, which requires a minimum of a 100 pg sample input. We used $270 \mathrm{pg}$ of RNA as the sample input and acquired a yield of $77.8 \mu \mathrm{g}$ of cRNA and $19.1 \mu \mathrm{g}$ of singlestrand cDNA (ss-cDNA). qPCR amplification of the cDNA for a housekeeping gene, $\beta$-actin, showed an amplicon at the expected molecular size on agarose gel electrophoresis (Fig. 4D). This result shows that the method for tissue processing and microdissection yields sufficient quality of RNA for downstream qPCR analysis. According to the manufacturer instruction, the cDNA yield we acquired is also sufficient for a potential application to perform microarray analysis (e.g., Whole-Transcriptome Array, Affymetrix). Using another sample of cDNA prepared in the same way, we confirmed that the successful laser capture collection of neurons by the amplification of the CaMKII $\alpha$ (for excitatory neurons) and DCX (for newborn neurons; Fig. 4D).

\section{Evaluation of RNA quality}

Our method uses conventional freezing, dehydration, laser capture, and RNA isolation procedures. There is no extra step to potentially reduce RNA quality, and therefore we expect that we can achieve the isolation of RNA in good quality, which is a requirement for the successful qPCR amplification described above. To confirm this expectation, we laser captured individual neurons in the granule cell layer one by one and pooled 5000 cells in each sample. Then we evaluated RNA integrity using Agilent RNA 6000 Pico Kit and 2100 Bioanalyzer (Schroeder et al., 2006). As described above, the yield of RNA collected from 100 to 300 cells was sufficient for successful downstream qPCR analysis but does not meet the minimum sample requirement for the RNA quality test. Therefore, we used larger samples by pooling 5000 cells for this analysis.

As shown in the electrophoresis images and electropherogram in Figure 5, two distinct bands of $18 \mathrm{~S}$ and $28 \mathrm{~S}$ ribosomal RNA were detected. RNA integrity numbers for two samples reached 7.5 and 7.2 (Schroeder et al., 2006). These values indicate a good quality of RNA, which is typically obtained by laser capture methods and is sufficient for downstream qPCR and transcriptomic applications (Butler et al., 2016; Ong et al., 2020). Thus, we validated that our hydration, laser capture, and RNA isolation method produce good-quality RNA.

\section{Specificity of microdissection}

The specificity for microdissection of single neuronal somata can be an issue since a targeted cell and its neighboring cells are often in close proximity. In addition, the typical thickness of brain sections (10-15 $\mu \mathrm{m})$ used for laser capture microdissection allows multiple neuronal soma overlap in z-direction. Therefore, a certain degree of contamination during the isolation is unavoidable. To evaluate specificity of microdissection, we examined YFP gene expression in visually identified $\mathrm{YFP}^{+}$neurons and YFP $^{-}$cells. For this experiment, we used POMC-ChR2- 

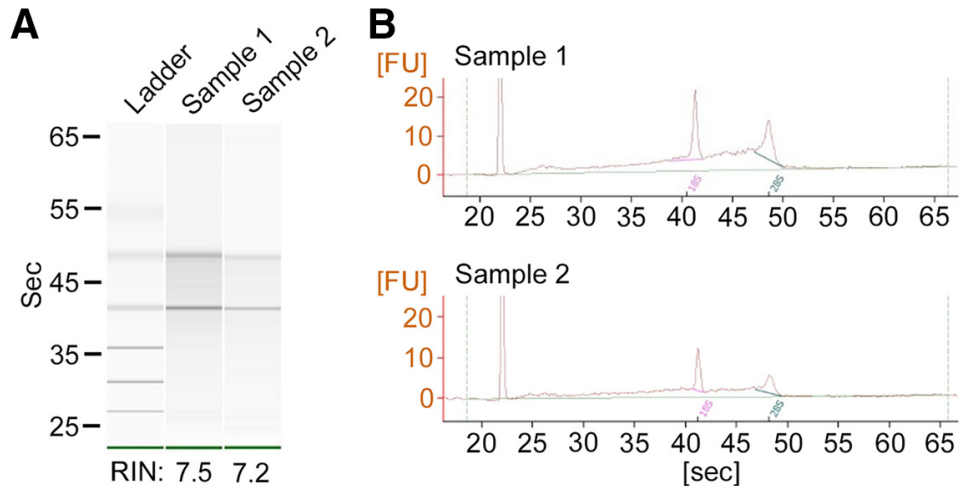

Figure 5. RNA quality assay. A, Electrophoresis images of RNA samples. Clear bands of $18 \mathrm{~S}$ and $28 \mathrm{~S}$ ribosomal RNAs are visible. Ladder: $0.2,0.5,1.0,2.0,4.0$, and $6.0 \mathrm{~kb}$. B. Electropherogram showing the distinct peaks of $18 \mathrm{~S}$ and $28 \mathrm{~S}$ ribosomal RNAs. FU, Fluorescence unit; Pink and thick green solid lines, baseline for $18 \mathrm{~S}$ and $20 \mathrm{~S}$ peaks, respectively; thin green solid line: overall baseline.

YFP mice that express ChR2-YFP in $\sim 10 \%$ of granule cells in the dentate gyrus. YFP ${ }^{+}$granule cells and neighboring $\mathrm{YFP}^{-}$cells were individually captured (Fig. 6A). The identity of YFP ${ }^{-}$cells was not confirmed, but most of them are likely granule cells because we selected them from the granule cell layer. Approximately 300 cells were pooled for each sample of $\mathrm{YFP}^{+}$and $\mathrm{YFP}^{-}$neurons. Collected RNA was reverse transcribed and amplified into cDNA with GeneChip WT Pico Kit. The amplification yield of $107 \mu \mathrm{g}\left(\mathrm{YFP}^{+}\right)$and $102 \mu \mathrm{g}\left(\mathrm{YFP}^{-}\right)$of CRNA and $16.9 \mu \mathrm{g}$ $\left(\mathrm{YFP}^{+}\right)$and $16.6 \mu \mathrm{g}\left(\mathrm{YFP}^{-}\right)$of ss-cDNA. From the amplified cDNA, the amount of YFP mRNA was quantified by qPCR. The result indicates there was as little as $10 \%$ of YFP mRNA in YFP ${ }^{-}$cells compared with the amount in neighboring $\mathrm{YFP}^{+}$granule cells (Fig. 6B), indicating high specificity in microdissection of single neuronal somata.

\section{Application of the technique to validate retrovirus- mediated single-cell gene knockout}

Virus-mediated expression of Cre recombinase has been used to achieve sparse, single-cell gene knockout (Tashiro et al., 2006a,b). Validation of such sparse gene knockout is not straightforward because unaffected wildtype cells are intermixed. We applied our method of laser capture microdissection with fluorescent proteins fused to transmembrane proteins to validate that such virusmediated single-cell gene knockout affects the expression of a target gene.

We used transgenic mice with the floxed NR1 gene, in which the expression of Cre recombinase results in the deletion of NR1 gene (also called GluN1) encoding a subunit of NMDA receptor (Tashiro et al., 2006a). We injected these mice with a bicistronic retroviral vector expressing YFP-fused enhanced halorhodopsin and Cre (RV-CAGeNpHR3.0-YFP-IRES-Cre) to knock out the NR1 gene. For control, transgenic mice were injected with a retroviral vector expressing YFP-fused enhanced halorhodopsin only. The vector constructs were described in Figure $7 \mathrm{~A}$. Brain sections were prepared $11 \mathrm{~d}$ after virus injection (Fig. 7B,C). For each sample, we captured $>100$ $\mathrm{YFP}^{+}$neurons and pooled them for RNA extraction. We prepared four samples each for control and NR1 knockout. Extracted RNA was reverse transcribed and amplified into cDNA with a GeneChip WT Pico Kit. The amplification yielded 63, 101, 203, and $197 \mu \mathrm{g}$ (control), and 62, 102, 178, and $210 \mu \mathrm{g}$ (NR1 knockout) of cRNA; and $14.4,15.2,14.4$, and $14.5 \mu \mathrm{g}$ (control), and 14, 15.6, 14.6 , and $14,5 \mu \mathrm{g}$ (NR1 knockout) of ss-cDNA. qPCR analysis showed a $92.2 \pm 11.0 \%$ reduction of the NR1 gene in the NR1 knock-out neurons compared with the control neurons (Fig. 7D,E), validating the successful removal of the NR1 gene in Cre-expressing granule cells.

\section{Discussion}

In this study, we demonstrated that genetic labeling with fluorescent proteins fused to transmembrane proteins allows for the selective collection of genetically labeled fluorescent neurons using laser capture microdissection. Fluorescent proteins fused to transmembrane proteins preserve fluorescence signals in sections prepared from freshly frozen brains after the standard tissue-processing procedure for laser capture microdissection. Stable fluorescence signals allowed us to distinguish neurons from non-neuronal cells and laser capture hundreds of neurons easily. We successfully isolated RNA from the collected neuronal somata and determined that $\sim 100$ cells are sufficient to obtain samples required for downstream applications such as QPCR and DNA microarray analysis.

Compared with conventional protocols of laser capture microdissection, observation and selection of fluorescently labeled cells are the only additional step. This additional step can be easily performed as far as a genetic method is available to label a cell type of interest with fluorescent proteins fused to transmembrane proteins. While this requirement may be a practical limitation for some applications, recent popularity in the use of optogenetic transmembrane proteins (Deisseroth et al., 2006; Pastrana, 2011; Pathak et al., 2013), which are often fused to fluorescent proteins, lowers a hurdle for this requirement. Plenty of transgenic and viral tools expressing channelrhodopsin or halorhodopsin are available commercially and/or from public depositories such as Addgene (http://www.addgene.org), which makes 
A

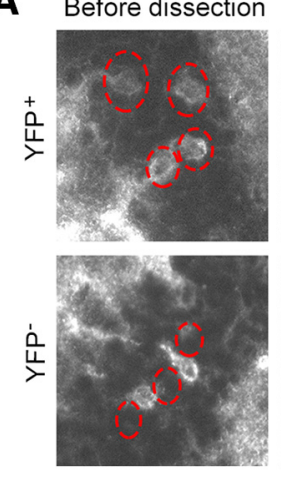

B

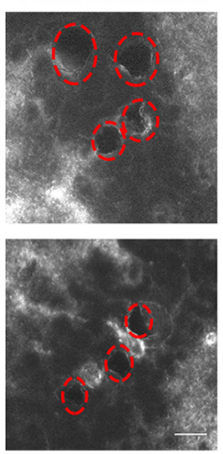

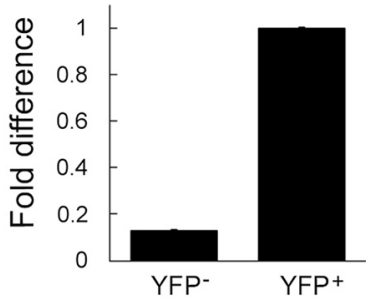

Figure 6. Evaluation of specific RNA collection from neurons targeted by laser capture microdissection. $\boldsymbol{A}$, Images of brain sections sparsely containing $\mathrm{YFP}^{+}$neurons in the dentate gyrus. The brain sections were prepared from Pomc-ChR2-YFP mice. YFP ${ }^{+}$neurons (top) and their neighboring $\mathrm{YFP}^{-}$cells (bottom) were visually selected for microdissection (red dotted ovals). Scale bar, $20 \mu \mathrm{m}$. $\boldsymbol{B}$, Relative amount of YFP RNA in $\mathrm{YFP}^{+}$neurons and their neighboring $\mathrm{YFP}^{-}$cells, quantified by qPCR analysis.

it relatively easy to express fluorescent proteins fused to transmembrane proteins in any neuronal (or other cell) types of interest.

We showed that fluorescent proteins fused to three different types of transmembrane proteins, ChR2, eNpHR, and TRPV2, can preserve fluorescence signals. ChR2 and TRPV2 are ion channels formed by multiple subunits with multiple transmembrane regions. eNpHR is an ion pump, but its structure is similar to that of ChR2. Thus, it is important to note that we tested relatively similar types of transmembrane proteins; therefore, it is not clear how much our result would be extended to other types of transmembrane proteins, for example, those with a single transmembrane domain.

We do not know why fluorescent proteins fused to those three transmembrane proteins can preserve fluorescence signals, but no regular or membrane-targeted protein does. However, it is known that freezethaw cycles damage the plasma membrane, and that soluble proteins, such as hemoglobin, are released from cells (Lovelock, 1953). Regular cytoplasmic fluorescent proteins are likely to diffuse out from cell bodies in this way. Transmembrane proteins are anchored tightly with and may stay with the plasma membrane, while the link with membrane-targeted fluorescent protein might not be strong enough to keep them associated with the membrane.

To validate the specificity of the RNA collection from selected cells, we used transgenic mice expressing ChR2-YFP and laser-captured $\mathrm{YFP}^{+}$and $\mathrm{YFP}^{-}$cells separately. qPCR analysis showed a 10-fold difference in the amount of YFP mRNA between $\mathrm{YFP}^{+}$and $\mathrm{YFP}^{-}$

A

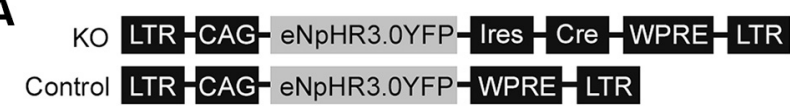

B

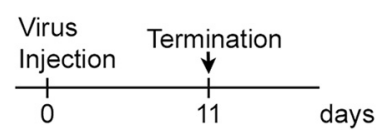

C

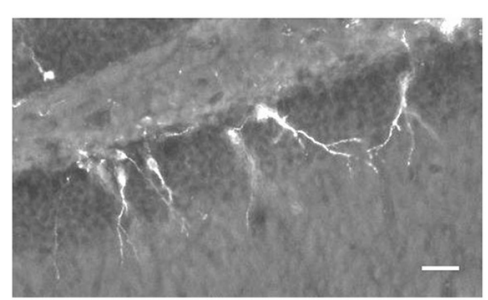

D

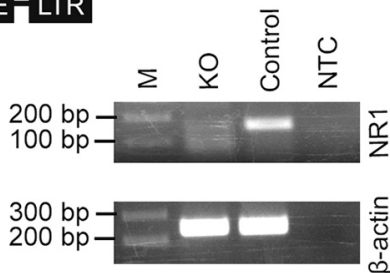

E

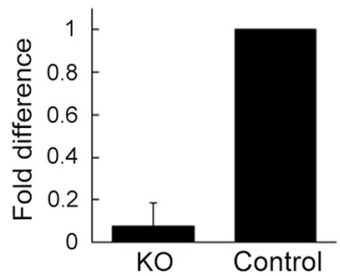

Figure 7. Validation of retrovirus-mediated, single-cell gene knockout in adult-born neurons in the dentate gyrus. $\boldsymbol{A}$, Schematic structures of the viral vector genomes. $\boldsymbol{B}$, Experimental time line. $\boldsymbol{C}$, Images of the dentate gyrus of floxed NR1 mice, which was injected with a bicistronic retroviral vector expressing eNpHR3.0-YFP and Cre. Scale bar, $50 \mu \mathrm{m}$. $\boldsymbol{D}$, qPCR amplification end products in gel electrophoresis. M, Molecular marker; KO, NR1 knock-out neurons with Cre expression; Control, control neurons without Cre expression; NTC, nontemplate control. E, qPCR quantification of NR1 RNA in Cre-expressing (KO) and control adult-born neurons. Note the $\sim 92 \%$ reduction in Cre-expressing neurons. 
cells, demonstrating the high specificity of RNA collection using this method, although a small amount of contamination from neighboring cells may not be avoidable.

We applied this method to demonstrate the effect of gene removal by viral expression of Cre recombinase in floxed NR1 mice. A bicistronic viral vector expressing eNpHR-YFP and Cre was injected into the dentate gyrus of adult mice, and we laser captured $\mathrm{YFP}^{+}$cells. The amount of NR1 mRNA in these $\mathrm{YFP}^{+}$cells was reduced by up to $92 \%$ compared with wild-type neurons. Thus, this experiment provides an example of successfully applying the technique to an actual experiment. This method would be useful for similar experimental needs in validating single-cell genetic manipulations (Zong et al., 2005; Judkewitz et al., 2009; Luo et al., 2016; Kaneko, 2017; Nishiyama et al., 2017).

One useful application of this method would be to examine gene expression in different neuronal types. This sort of gene expression study has been successfully performed using genetic labeling with regular cytoplasmic fluorescent proteins and fluorescent-based sorting. However, genetic labeling often marks multiple neuronal types and/or other cell types (van Praag et al., 2002; Balthasar et al., 2004; Lagace et al., 2007; Harris et al., 2014), which makes it difficult to achieve RNA collection from a single neuronal type. For example, while the expression of parvalbumin is often used to genetically define a subtype of interneurons, it is well known that parvalbuminexpressing interneurons contain multiple neuronal types defined morphologically, including basket cells and axo-axonic cells (Hu et al., 2014; Nassar et al., 2015). In the conventional fluorescence-based sorting, these neuronal types cannot be separated. However, using our method, one should be able to separately laser capture these two morphologically distinct neuronal types and to analyze the gene expression of each neuronal type. One caveat for this application is a requirement of relatively sparse labeling to allow for visualization of the dendrites of individual neurons. For example, in Pomc-ChR2-YFP mice that we used in this study, $\sim 10 \%$ of granule cells express ChR2-YFP (Uemura et al., 2021), and we can clearly observe their dendrites (Fig. 2C). However, in another line of Pomc-ChR2-YFP mice that we used in a different study, nearly all granule cells express ChR2-YFP, the whole molecular layer of the dentate gyrus, which is occupied by granule cell dendrites, are filled with YFP fluorescence (Âmellem et al., 2021). In this case, we cannot visualize the dendritic morphology of individual neurons. Therefore, not all fluorescence-labeling methods work for this approach, and one may need to select a method for relatively sparse labeling (Zong et al., 2005; Badea et al., 2009; Judkewitz et al., 2009; Luo et al., 2016; Kaneko, 2017; Nishiyama et al., 2017).

Some morphological classification of neuronal types is largely subjective, which may make it difficult to reach agreement on classification among individual researchers. Or even if they are visually clearly distinguishable, there is no common ground for judging whether they should be classified as separate neuronal types. We believe that our method could provide a solution for this issue by giving an independent rationale for such "subjective" classification criteria. For this purpose, one needs to apply a subjective morphological classification and to perform gene expression analysis of those neuronal types using our method. If the two neuronal types have a clear difference in gene expression, one can conclude that they are two different neuronal types. If they do not have a difference in gene expression, one can conclude that they may not be different neuronal types. Furthermore, if some genes are expressed only in one of two neuronal types, one can use them as a marker for the neuronal type, which would make it easier to further confirm and use the classification in future studies. When it is difficult to make clear morphological criteria, one can test multiple subjective criteria. When the criteria separate two neuronal types better, the difference in gene expression would be clearer. Based on this idea, one determines the best version of morphological classification in terms of a difference in gene expression. Our method should be helpful to provide such tentative classification of neuronal subtypes based on morphology with an independent basis/rationale in terms of gene expression.

Related to the importance of morphological visualization, fluorescent proteins fused to transmembrane have an additional advantage. Compared with cytosolic fluorescent proteins, fluorescent proteins fused to transmembrane fluorescence proteins are distributed along cellular membranes and visualize small processes better than cytosolic fluorescent proteins (Moriyoshi et al., 1996; Fig. $2 B$ ). Such clearer visualization of dendritic morphology facilitates the identification of neuronal types.

In addition to gene expression profiling, laser capture collection of a selective neuronal population may be applied to different types of genomic analyses. Such applications include chromatin immunoprecipitation sequencing (seq) to identify binding sites for DNA-binding proteins (Park, 2009), the assay for transposase-accessible chromatin-seq to map chromatin accessibility (Buenrostro et al., 2013), and ChIA$\mathrm{PET}, \mathrm{Hi}-\mathrm{C}$, and HiChip to examine tridimensional chromatin conformation (Fullwood and Ruan, 2009; van Berkum et al., 2010; Mumbach et al., 2016).

Our study demonstrated the stability of fluorescence signals of fluorescent proteins fused to transmembrane proteins, which allows for selectively collecting a cell type of interest with laser capture microdissection. We validated that the RNA sample isolated with this method is suitable for downstream gene expression analysis experiments such as qPCR. The isolation of single neurons is specific with minimum contamination from neighboring cells. This methodology provides a novel alternative approach for gene expression analysis targeting a specific neuronal/cell type, which is especially advantageous when the specificity of genetic labeling is not sufficient and additional selection based on morphology is required.

\section{References}

Åmellem I, Yovianto G, Chong HT, Nair RR, Cnops V, Thanawalla A, Tashiro A (2021) Role of NMDA receptors in adult neurogenesis and normal development of the dentate gyrus. eNeuro 8: ENEURO.0566-20.2021. 
Badea TC, Hua ZL, Smallwood PM, Williams J, Rotolo T, Ye X, Nathans $J$ (2009) New mouse lines for the analysis of neuronal morphology using CreER(T)/loxP-directed sparse labelling. PLoS One 4:e7859.

Balthasar N, Coppari R, McMinn J, Liu SM, Lee CE, Tang V, Kenny CD, McGovern RA, Chua SC Jr, Elmquist JK, Lowell BB (2004) Leptin receptor signaling in POMC neurons is required for normal body weight homeostasis. Neuron 42:983-991.

Bonner RF, Emmert-Buck M, Cole K, Pohida T, Chuaqui R, Goldstein S, Liotta LA (1997) Laser capture microdissection: molecular analysis of tissue. Science 278:1481, 1483.

Buenrostro JD, Giresi PG, Zaba LC, Chang HY, Greenleaf WJ (2013) Transposition of native chromatin for fast and sensitive epigenomic profiling of open chromatin, DNA-binding proteins and nucleosome position. Nat Methods 10:1213-1218.

Butler AE, Matveyenko AV, Kirakossian D, Park J, Gurlo T, Butler PC (2016) Recovery of high-quality RNA from laser capture microdissected human and rodent pancreas. J Histotechnol 39:59-65.

Chatzi C, Zhang Y, Hendricks WD, Chen Y, Schnell E, Goodman RH, Westbrook GL (2019) Exercise-induced enhancement of synaptic function triggered by the inverse BAR protein, Mtss1L. Elife 8: e45920.

Daigle TL, Madisen L, Hage TA, Valley MT, Knoblich U, Larsen RS, Takeno MM, Huang L, Gu H, Larsen R, Mills M, Bosma-Moody A, Siverts LA, Walker M, Graybuck LT, Yao Z, Fong O, Nguyen TN, Garren E, Lenz GH, et al. (2018) A suite of transgenic driver and reporter mouse lines with enhanced brain-cell-type targeting and functionality. Cell 174:465-480.e22.

Deisseroth K, Feng G, Majewska AK, Miesenböck G, Ting A, Schnitzer MJ (2006) Next-generation optical technologies for illuminating genetically targeted brain circuits. J Neurosci 26:1038010386.

Emmert-Buck MR, Bonner RF, Smith PD, Chuaqui RF, Zhuang Z, Goldstein SR, Weiss RA, Liotta LA (1996) Laser capture microdissection. Science 274:998-1001.

Fend F, Raffeld M (2000) Laser capture microdissection in pathology. J Clin Pathol 53:666-672.

Fullwood MJ, Ruan Y (2009) ChIP-based methods for the identification of long-range chromatin interactions. J Cell Biochem 107:3039

Harris JA, Hirokawa KE, Sorensen SA, Gu H, Mills M, Ng LL, Bohn P, Mortrud M, Ouellette B, Kidney J, Smith KA, Dang C, Sunkin S, Bernard A, Oh SW, Madisen L, Zeng H (2014) Anatomical characterization of Cre driver mice for neural circuit mapping and manipulation. Front Neural Circuits 8:76.

Ho H, Both M, Siniard A, Sharma S, Notwell JH, Wallace M, Leone DP, Nguyen A, Zhao E, Lee $H$, Zwilling D, Thompson KR, Braithwaite SP, Huentelman M, Portmann T (2018) A guide to single-cell transcriptomics in adult rodent brain: the medium spiny neuron transcriptome revisited. Front Cell Neurosci 12:159.

Hu H, Gan J, Jonas P (2014) Interneurons. Fast-spiking, parvalbumin ${ }^{+}$ GABAergic interneurons: from cellular design to microcircuit function. Science 345:1255263.

Hussain RZ, Miller-Little WA, Doelger R, Cutter GR, Loof N, Cravens PD, Stüve O (2018) Defining standard enzymatic dissociation methods for individual brains and spinal cords in EAE. Neurol Neuroimmunol Neuroinflamm 5:e437.

Jiang W, Hunter T (1998) Analysis of cell-cycle profiles in transfected cells using a membrane-targeted GFP. Biotechniques 24:348354.

Judkewitz B, Rizzi M, Kitamura K, Häusser M (2009) Targeted singlecell electroporation of mammalian neurons in vivo. Nat Protoc 4:862-869.

Kaneko T (2017) Genome editing in mouse and rat by electroporation. In: Genome editing in animals (Hatada I, eds), pp 163081163089. New York: Springer.

Kalejta RF, Shenk T, Beavis AJ (1997) Use of a membrane-localized green fluorescent protein allows simultaneous identification of transfected cells and cell cycle analysis by flow cytometry. Cytometry 29:286-291.
Kitanishi T, Ujita S, Fallahnezhad M, Kitanishi N, Ikegaya Y, Tashiro A (2015) Novelty-induced phase-locked firing to slow gamma oscillations in the hippocampus: requirement of synaptic plasticity. Neuron 86:1265-1276.

Lagace DC, Whitman MC, Noonan MA, Ables JL, DeCarolis NA, Arguello AA, Donovan MH, Fischer SJ, Farnbauch LA, Beech RD, DiLeone RJ, Greer CA, Mandyam CD, Eisch AJ (2007) Dynamic contribution of nestin-expressing stem cells to adult neurogenesis. J Neurosci 27:12623-12629.

Lovelock JE (1953) The haemolysis of human red blood-cells by freezing and thawing. Biochim Biophys Acta 10:414-426.

Luo W, Mizuno H, Iwata R, Nakazawa S, Yasuda K, Itohara S, Iwasato T (2016) Supernova: a versatile vector system for single-cell labeling and gene function studies in vivo. Sci Rep 6:35747.

Madisen L, Mao T, Koch H, Zhuo JM, Berenyi A, Fujisawa S, Hsu YW, Garcia AJ, Gu X, Zanella S, Kidney J, Gu H, Mao Y, Hooks BM, Boyden ES, Buzsáki G, Ramirez JM, Jones AR, Svoboda K, Han X, et al. (2012) A toolbox of Cre-dependent optogenetic transgenic mice for light-induced activation and silencing. Nat Neurosci 15:793-802.

Moriyoshi K, Richards LJ, Akazawa C, O'Leary DD, Nakanishi S (1996) Labeling neural cells using adenoviral gene transfer of membrane-targeted GFP. Neuron 16:255-260.

Mumbach MR, Rubin AJ, Flynn RA, Dai C, Khavari PA, Greenleaf WJ, Chang HY (2016) HiChIP: efficient and sensitive analysis of protein-directed genome architecture. Nat Methods 13:919922.

Nassar M, Simonnet J, Lofredi R, Cohen I, Savary E, Yanagawa Y, Miles R, Fricker D (2015) Diversity and overlap of parvalbumin and somatostatin expressing interneurons in mouse presubiculum. Front Neural Circuits 9:20.

Nishiyama J, Mikuni T, Yasuda R (2017) Virus-mediated genome editing via homology-directed repair in mitotic and postmitotic cells in mammalian brain. Neuron 96:755-768.

Ong CJ, Tan QX, Lim HJ, Shannon NB, Lim WK, Hendrikson J, Ng WH, Tan JWS, Koh KKN, Wasudevan SD, Ng CCY, Rajasegaran V, Lim TKH, Ong CK, Kon OL, Teh BT, Tan GHC, Chia CS, Soo KC, Teo MCC (2020) An optimised protocol harnessing laser capture microdissection for transcriptomic analysis on matched primary and metastatic colorectal tumours. Sci Rep 10:682.

Park PJ (2009) ChIP-seq: advantages and challenges of a maturing technology. Nat Rev Genet 10:669-680.

Pastrana E (2011) Optogenetics: controlling cell function with light. Nat Methods 8:24-25.

Pathak GP, Vrana JD, Tucker CL (2013) Optogenetic control of cell function using engineered photoreceptors. Biol Cell 105:59-72.

Reichard A, Asosingh K (2019) Best practices for preparing a single cell suspension from solid tissues for flow cytometry. Cytometry A 95:219-226.

Rossner MJ, Hirrlinger J, Wichert SP, Boehm C, Newrzella D, Hiemisch H, Eisenhardt G, Stuenkel C, von Ahsen O, Nave KA (2006) Global transcriptome analysis of genetically identified neurons in the adult cortex. J Neurosci 26:9956-9966.

Schroeder A, Mueller O, Stocker S, Salowsky R, Leiber M, Gassmann G, Lightfoot S, Menzel W, Granzow M, Ragg T (2006) The RIN: an RNA integrity number for assigning integrity values to RNA measurements. BMC Mol Biol 7:3.

Singh S, Wang L, Schaff DL, Sutcliffe MD, Koeppel AF, Kim J, Onengut-Gumuscu S, Park K-S, Zong H, Janes KA (2019) In situ 10-cell RNA sequencing in tissue and tumor biopsy samples. Sci Rep 9:4836.

Tashiro A, Sandler VM, Toni N, Zhao C, Gage FH (2006a) NMDA-receptor-mediated, cell-specific integration of new neurons in adult dentate gyrus. Nature 442:929-933.

Tashiro A, Zhao C, Gage F (2006b) Retrovirus-mediated single-cell gene knockout technique in adult newborn neurons in vivo. Nat Protoc 1:3049-3055.

Tashiro A, Zhao C, Suh H, Gage FH (2015a) Purification and injection of retroviral vectors. Cold Spring Harb Protoc 2015:925-931. 
Tashiro A, Zhao C, Suh H, Gage FH (2015b) Imaging newborn granule cells in fixed sections. Cold Spring Harb Protoc 2015:932-933.

Tsien JZ, Huerta PT, Tonegawa S (1996) The essential role of hippocampal CA1 NMDA receptor-dependent synaptic plasticity in spatial memory. Cell 87:1327-1338.

Uemura M, Blankvoort S, Tok SSL, Yuan L, Cobar LF, Lit KK, Tashiro A (2021) A neurogenic microenvironment defined by excitatory-inhibitory neuronal circuits in adult dentate gyrus. Cell Rep 36:109324.

van Berkum NL, Lieberman-Aiden E, Williams L, Imakaev M, Gnirke A, Mirny LA, Dekker J, Lander ES (2010) Hi-C: a method to study the three-dimensional architecture of genomes. J Vis Exp 39: e1869. van Praag H, Schinder AF, Christie BR, Toni N, Palmer TD, Gage FH (2002) Functional neurogenesis in the adult hippocampus. Nature 415:1030-1034.

Xia N, Fang F, Zhang P, Cui J, Tep-Cullison C, Hamerley T, Lee HJ, Palmer T, Bothner B, Lee JH, Pera RR (2017) A knockin reporter allows purification and characterization of mDA neurons from heterogeneous populations. Cell Rep 18:2533-2546.

Zhao C, Teng EM, Summers RG, Ming GL, Gage FH (2006) Distinct morphological stages of dentate granule neuron maturation in the adult mouse hippocampus. J Neurosci 26:3-11.

Zong H, Espinosa JS, Su HH, Muzumdar MD, Luo L (2005) Mosaic analysis with double markers in mice. Cell 121:479-492. 